\title{
RESISTÊNCIA DA PLANTA DANINHA CAPIM-MARMELADA (Brachiaria plantaginea) AOS HERBICIDAS INIBIDORES DA ENZIMA ACCASe NA CULTURA DA SOJA ${ }^{1}$
}

\author{
DIONISIO L. P. GAZZIERO ${ }^{2}$, PEDRO J. CHRISTOFFOLETI ${ }^{3}$, ALEXANDRE M. BRIGHENTI ${ }^{2}$, CÁSSIO E. C. PRETE ${ }^{4}$ e \\ ELEMAR VOLL ${ }^{2}$
}

\section{RESUMO}

Populações de capim-marmelada (Brachiaria plantaginea) infestantes da cultura da soja (Glycine $\max ($ L.) Merrill) no município de Mangueirinha, PR, controlada, ano após ano, com herbicidas inibidores da ACCase, apresentaram falhas de controle quando esses produtos foram aplicados na safra 95/96. Experimentos de doseresposta foram conduzidos em condições de casa de vegetação do Departamento de Horticultura da Escola Superior de Agricultura "Luiz de Queiroz", Piracicaba, SP, e da Embrapa Soja, Londrina, PR, com o objetivo de confirmar essa resistência, bem como avaliar o nível de resistência e resistência cruzada a herbicidas pertencentes aos grupos químicos das ciclohexanidionas e ariloxifenoxi-propionato. Plantas de capimmarmelada supostamente resistentes foram tratadas com diversos herbicidas e doses e comparadas com plantas de uma população suscetível dessa infestante. Os tratamentos foram estabelecidos considerando-se as doses recomendadas dos produtos, metade delas e, duas, quatro e oito vezes superiores a recomendada. Os produtos e as doses aplicadas foram haloxyfopmethyl nas doses $0 ; 60 ; 120 ; 240 ; 480$ e $960 \mathrm{~g}$ i.a./ha, mais o espalhante adesivo Joint $0,5 \%$ v/v, fluazifop-p-butil nas doses $0 ; 94 ; 188 ; 376$; 752 e 1504 gi.a./ha, mais o espalhante adesivo
Agral $0,2 \% \mathrm{v} / \mathrm{v}$, sethoxydim nas doses $0 ; 115$; 230; 460; 920 e 1840 g. Os resultados de percentagem de controle foram submetidos à análise de regressão e, através dos modelos ajustados, foram obtidos os valores de $\mathrm{GR}_{50}$ (dose necessária para proporcionar $50 \%$ de controle de cada biótipo). As relações médias de $\mathrm{GR}_{50}$ e os diferenciais de controle nas doses recomendadas dos herbicidas (S-R) foram calculados. Os diferenciais de controle nas doses recomendadas (S-R) foram 97 e 11; 96 e 62; 99 e 86; 0,6 e 4; 20 e 17; 88 e 35 para os herbicidas haloxyfop-methyl, fluazifop-p-butil, sethoxydim, clethodim, propaquizafop e fenoxaprop-p-ethyl nos experimentos conduzidos em Piracicaba e Londrina, respectivamente. Conclui-se que o biótipo B-196 é resistente aos herbicidas inibidores da ACCase e apresenta diferentes níveis de resistência cruzada aos herbicidas estudados, exceto clethodim. Os herbicidas sethoxydim, fluazifop-p-butil, haloxyfop-methyl e fenoxaprop-ethyl são os produtos para os quais o biótipo apresentou maior grau de resistência quando comparado com clethodim e propaquizafop.

Palavras chave: Papuã, graminicidas, controle químico, experimentos de dose-resposta.

\footnotetext{
${ }^{1}$ Recebido para publicação em 04/02/98 e na forma revisada em 21/06/98.

$2 \mathrm{Eng}^{\circ} \mathrm{Agr}^{\circ}$ Pesquisador, Embrapa Soja. C.P. 231, CEP: $86001-970$, Londrina/PR.

${ }^{3}$ Prof $^{\circ}$ Dr., Dept ${ }^{\circ}$ de Horticultura da ESALQ/USP. C.P. 9, CEP: 13418-900, Piracicaba/SP.

${ }^{4}$ Prof $^{\circ}$ Dr., Dept ${ }^{\circ}$ de Fitotecnia da UEL. C.P. 6001, CEP: 86051-990, Londrina/PR.
} 


\section{ABSTRACT \\ Alexandergrass (Brachiaria plantaginea) resistance to ACCase inhibitor herbicides}

Alexandergrass population in Mangueirinha County, Paraná State, Brazil, was controled by ACCase inhibitor herbicides for more than ten years. However, no control was observed in soybean season 95/96. Dose-response experiments were caried out in greenhouses of Horticulture Department-ESALQ/USP and Embrapa Soja in order to study the level of resistance and cross resistance to aryloxiphenoxypropionic and cyclohexanodione herbicides. The supposedly resistant biotype (B196) and susceptible biotype were treated with ACCase inhibitors at rates corresponding to $0,1 / 2$, $1,2,4$ and 8 fold the recommended rate. The herbicides and their recommended doses were haloxyfop-methyl (120 g/ha), fluazifop-p-butil (188 g/ha), sethoxydim (230 g/ha), clethodim (108 g/ha), propaquizafop (125 g/ha) and fenoxaprop-p-ethyl ( $82 \mathrm{~g} / \mathrm{ha})$. The adjuvants were added following the recommendation of each herbicide. It was estimated the $\mathrm{GR}_{50}$ values, $\mathrm{R} / \mathrm{S}$ ratios and $\mathrm{S}-\mathrm{R}$ (the difference between control percentage of both biotypes at recomendated rates). The results of resistance level among the biotypes and herbicides showed differences, with variable $\mathrm{GR}_{50}$ values for herbicides and biotypes. The S-R values were 97 and 11; 96 and 62; 99 and 86; 0.6 and 4; 20 and 17;88 and 35 for the herbicides haloxyfop-methyl, fluazifop-p-butil, sethoxydim, clethodim, propaquizafop and fenoxaprop-ethyl in Piracicaba and Londrina, respectively. The Brachiaria plantaginea biotype B-196 is resistant to ACCase inhibitor herbicides and exhibited different levels of cross resistance to the herbicides, except clethodim. Sethoxydim, fluazifop-p-butil, haloxyfop-methyl and fenoxaprop-ethyl were the herbicides which the biotype showed high levels of resistance compared to clethodim and propaquizafop.

Key words: Alexanderweed, graminicides, chemical control, doses response curve.

\section{INTRODUÇÃO}

O uso extensivo e persistente de herbicidas como o único método de controle de plantas daninhas ocasiona o aparecimento de populações resistentes aos mesmos. Na maior parte das vezes, essa resistência surge em sistemas de monocultura onde um único herbicida é aplicado continuamente (Maxwell et al., 1990; Blair, 1991).

A resistência pode ser definida como uma condição onde as plantas daninhas sobrevivem às doses normalmente empregadas no campo, como resultado da seleção ou da resposta genética às repetidas exposições aos herbicidas de mesmo mecanismo/local de ação. Outras definições importantes são resistência cruzada e resistência múltipla. $\mathrm{O}$ primeiro caso ocorre quando a expressão de um mecanismo de resistência faz com que uma população de plantas daninhas se torne resistente aos herbicidas de diferentes classes químicas, porém com o mesmo mecanismo de ação. A forma mais comum de resistência cruzada é quando uma mudança no sítio de ação de um herbicida também confere, resistência aos herbicidas de diferentes classes que inibem o mesmo sítio de ação. A resistência múltipla ocorre quando as plantas possuem dois ou mais mecanismos de resistência distintos ( Powles \& Preston, 1995).

A primeira menção de um possível caso de resistência de plantas daninhas a herbicidas foi observado em 1957, com o produto 2,4-D (Whitehead \& Switzer, 1962). Em 1970, foi relatada a resistência à atrazine (Ryan, 1970) e, em 1973, à trifluralin (Mudge et al., 1984). Populações de Bidens pilosa, Brachiaria plantaginea e Euphorbia heterophylla resistentes aos herbicidas foram confirmadas em algumas regiões produtoras de soja no Brasil e relatadas 
por Vidal \& Fleck (1997); Vidal et al., 1997a; Vidal et al., 1997b. A existência de populações resistentes de Bidens pilosa aos herbicidas inibidores da enzima ALS foram confirmadas em lavouras de soja no Brasil por Christoffoleti et al. (1996). Nos estados do Mato Grosso do Sul e do Rio Grande do Sul, foram identificadas populações de picão-preto resistentes aos herbicidas dessa mesma classe Ponchio (1997). A resistência de um biótipo da planta daninha Brachiaria plantaginea aos herbicidas inibidores da enzima ACCase (acetil CoA carboxilase), provenientes de uma área de cultura de soja da região sudoeste do Estado do Paraná, foi confirmada por Gazziero et al.(1997). Christoffoleti et al. (1998) também confirmaram a resistência de populações de capim-marmelada no Estado do Paraná aos herbicidas inibidores da ACCase.

Recentemente, têm-se observado diversos campos de produção de soja no Brasil com falhas de controle do capim-marmelada pelos herbicidas inibidores da enzima ACCase recomendados para essa cultura. Nessa situação, tem-se atribuído como causa dessas falhas o desenvolvimento de biótipos resistentes sem nenhuma comprovação científica.

Sendo assim, este experimento foi conduzido com os objetivos de confirmar a resistência aos herbicidas inibidores da ACCase de um biótipo (B-196) de capim-marmelada e avaliar o nível de resistência cruzada aos herbicidas pertencentes a diferentes grupos químicos.

\section{MATERIAL E MÉTODOS}

Dois experimentos foram conduzidos em casa-de-vegetação, sendo um deles, instalado no Departamento de Horticultura da Escola Superior de Agricultura "Luiz de Queiroz" - Universidade de São Paulo, Piracicaba, SP, no mês de novembro de 1996. O outro, foi conduzido, no mesmo período, na Embrapa Soja, Londrina, PR. As sementes de capim-marmelada foram coletadas em uma área do município de Mangueirinha, sudoeste do Estado do Paraná, onde suspeitava-se do desenvolvimento da resistência, denominando o biótipo de B-196. Essa área foi pulverizada durante vários anos com herbicidas inibidores da enzima ACCase e, nos últimos anos, foram observados escapes dessa planta daninha a esses herbicidas. Amostras de sementes de capimmarmelada, consideradas como suscetível, pois não recebiam tratamentos com herbicidas inibidores da enzima ACCase, foram coletadas nas respectivas regiões de condução dos experimentos.

Os experimentos foram conduzidos em delineamento experimental em blocos casualizados com quatro repetições. Os tratamentos foram estabelecidos considerando-se as doses recomendadas dos herbicidas, metade delas e, duas, quatro e oito vezes superiores as recomendadas, além da testemunha (dose zero). Os produtos e as doses recomendadas foram haloxyfop-methyl (120 g/ha), fluazifop-p-butil (188 g/ha), sethoxydim (230 g/ha), clethodim (108 g/ha), propaquizafop (125 g/ha), e fenoxaprop-p-ethyl (82 g/ha). Foram adicionados os adjuvantes de acordo com as especificações dos fabricantes dos herbicidas.

No experimento realizado em Piracicaba, foi semeada, em cada vaso, uma grande quantidade de sementes para posterior desbaste, não sendo utilizado nenhum procedimento de quebra da dormência. Entretanto, no experimento conduzido em Londrina, todas as sementes foram submetidas ao tratamento com ácido sulfúrico, durante 5 minutos, para quebra da dormência.

Sementes do biótipo considerado suscetível e daquele onde suspeitava-se da resistência, foram semeadas separadamente em vasos plásticos de 2,0 1 de capacidade, para o experimento realizado em Piracicaba, e em vasos de $500 \mathrm{ml}$ para o experimento de Londrina, totalizando 288 vasos em cada local. O material utilizado para o enchimento dos vasos foi substrato, composto da mistura de três partes de solo para uma de húmus, 
em ambos os experimentos. A análise química dos materiais dos substratos encontra-se na Tabela 1.

Os experimentos foram mantidos em casa-de-vegetação, sendo os vasos irrigados regularmente, mantendo-se a umidade do substrato próxima à capacidade de campo. Após a emergência, foi feito o desbaste, deixando-se cinco plântulas por vaso.

Em Piracicaba, a aplicação dos herbicidas ocorreu no $20^{\circ}$ dia após a emergência (DAE), quando o capim-marmelada encontrava-se com quatro folhas. Foi utilizada câmara de pulverização especialmente projetada para aplicação de herbicidas em vasos, onde a calda é pressurizada por ar comprimido. O volume de aplicação foi de 300 1/ha. A barra de pulverização continha um bico com ponta $110.03 \mathrm{E}$, de jato leque e uniforme à pressão de trabalho de $207 \mathrm{kPa}$. A temperatura do ar durante a aplicação foi de $28^{\circ} \mathrm{C}$, com umidade relativa de $60 \%$.

Em Londrina, a aplicação dos herbicidas foi realizada no $21^{\circ}$ DAE, quando o capimmarmelada apresentava-se com quatro a seis folhas. Utilizou-se pulverizador costal, à pressão constante (mantida por $\mathrm{CO}_{2}$ comprimido) de $241 \mathrm{kPa}$, equipado com barra de 1,0 m de largura e dois bicos de jato plano (tipo leque) 110 D33 (Micron Porcelane), distanciados 0,5 m, com volume de pulverização equivalente a $220 \mathrm{l} / \mathrm{ha}$. A temperatura do ar durante a aplicação foi de $26^{\circ} \mathrm{C}$, com umidade relativa de $88 \%$.

$\mathrm{O}$ efeito dos herbicidas no controle de plantas de capim-marmelada foi avaliado aos $14 \mathrm{e}$ aos 10 dias após a aplicação dos produtos para os experimentos conduzidos em Piracicaba e Londrina, respectivamente. Utilizou-se a escala visual de $0-100 \%$ onde $0 \%$ (zero) representava nenhum controle e $100 \%$ o controle total.

Foram ajustados modelos de regressão aos dados observados, tendo como variável resposta a percentagem de controle da planta daninha e como variável explanatória doses de herbicidas. Os dados foram transformados em arco seno $\sqrt{x / 100}$. Os valores de $\mathrm{GR}_{50}$ (dose necessária para proporcionar $50 \%$ de controle do biótipo resistente (R) e do suscetível (S)) foram obtidos a partir dos modelos ajustados. Em seguida, calculou-se as relações médias de $\mathrm{GR}_{50}$ para cada produto, dividindo-se o $\mathrm{GR}_{50}$ do biótipo resistente pelo do biótipo suscetível. O valor encontrado expressa o número de vezes em que a dose necessária para controlar $50 \%$ das plantas do biótipo suscetível deve ser superior para que possa ocorrer o mesmo efeito com o biótipo resistente. Foram obtidos, ainda, os diferenciais de controle na dose recomendada (S-R), subtraindo-se a percentagem de controle do biótipo suscetível da do resistente.

Todo material vegetal e sementes restantes dos testes foram eliminados para evitar disseminação involuntária do biótipo resistente.

\section{RESULTADOS E DISCUSSÃO}

O herbicida haloxyfop-methyl proporcionou uma percentagem média de controle de $1 \%$ para o capim-marmelada biótipo B-196 quando se utilizou a dose recomendada do produto; entretanto, essa mesma dose proporcionou uma percentagem de controle de 98\% para o biótipo suscetível no experimento conduzido em Piracicaba (Tabela 2). Em Londrina, houve um controle acima de $80 \%$ com a dose recomendada do produto para ambos os biótipos estudados. Provavelmente, o controle obtido no experimento de Londrina tenha sido melhor do que o alcançado no experimento de Piracicaba, devido às plantas estarem mais vulneráveis ao produto em função das condições a que foram submetidas, ou seja, menor volume de solo a ser explorado pelas plantas, e também, às próprias condições climáticas da região. 
TABELA 1. Resultado da análise química dos substratos utilizados para o crescimento das plantas, no experimento conduzido em Piracicaba e Londrina.

\begin{tabular}{lccccccc}
\hline Locais & $\begin{array}{c}\mathrm{P} \\
-\mathrm{gH} / \mathrm{dm}^{3}--\end{array}$ & $\mathrm{CaCl}_{2}$ & $\mathrm{Al}$ & $\mathrm{K}$ & $\begin{array}{c}\mathrm{Ca} \\
\end{array}$ & $\mathrm{Mg}$ & $\mathrm{H}+\mathrm{Al}$ \\
\hline Piracicaba $^{1}$ & 168.0 & 5.60 & 0.0 & 2.55 & 6.7 & 3.20 & 2,00 \\
Londrina $^{2}$ & 86.7 & 6.02 & 0.0 & 2.13 & 7.05 & 2.53 & 3.19 \\
\hline
\end{tabular}

1. Resultados fornecidos pelo laboratório de análises de solos da ESALQ/USP.

2. Resultados fornecidos pelo laboratório de análises de solos da Embrapa Soja

TABELA 2. Percentagem média de controle do capim-marmelada (Brachiaria plantaginea), biótipo resistente (R) e suscetível (S) em função das doses dos herbicidas para o experimento de Piracicaba (P) e Londrina (L).

\begin{tabular}{|c|c|c|c|c|c|c|c|c|c|c|c|c|}
\hline \multirow{3}{*}{ Doses* } & \multicolumn{4}{|c|}{ Haloxyfop-methyl } & \multicolumn{4}{|c|}{ Fluazifop - p- butil } & \multicolumn{4}{|c|}{ Sethoxydim } \\
\hline & \multicolumn{2}{|c|}{$\mathrm{P}$} & \multicolumn{2}{|c|}{$\mathrm{L}$} & \multicolumn{2}{|c|}{$\mathrm{P}$} & \multicolumn{2}{|c|}{$\mathrm{L}$} & \multicolumn{2}{|c|}{$\mathrm{P}$} & \multicolumn{2}{|c|}{$\mathrm{L}$} \\
\hline & $\mathrm{R}$ & $S$ & $\mathrm{R}$ & $S$ & $\mathrm{R}$ & $\bar{S}$ & $\mathrm{R}$ & $S$ & $\mathrm{R}$ & $\mathrm{S}$ & $\mathrm{R}$ & $\mathrm{S}$ \\
\hline $0,5 \mathrm{X}$ & 0,00 & 90,00 & 60,00 & 78,25 & 0,0 & 97,50 & 13,75 & 72,50 & 0,0 & 95,0 & 5,0 & 71,25 \\
\hline $1,0 X$ & 1,25 & 98,75 & 80,00 & 91,25 & 0,0 & 96,25 & 21,25 & 83,75 & 0,0 & 99,0 & 5,0 & 91,25 \\
\hline $2,0 \times$ & 38,75 & 100,0 & 78,75 & 95,00 & 35,0 & 97,50 & 50,00 & 92,50 & 0,0 & 100,0 & 10,0 & 100,0 \\
\hline $4,0 \times$ & 87,50 & 100,0 & 95,00 & 100,0 & 50,0 & 100,0 & 55,00 & 100,0 & 0,0 & 100,0 & 20,0 & 100,0 \\
\hline $8,0 \mathrm{X}$ & 92,50 & 100,0 & 96,25 & 100,0 & 55,0 & 100,0 & 83,75 & 100,0 & 5,0 & 100,0 & 51,2 & 100,0 \\
\hline \multirow{3}{*}{ Doses* } & \multicolumn{4}{|c|}{ Clethodim } & \multicolumn{4}{|c|}{ Propaquizafop } & \multicolumn{4}{|c|}{ Fenoxaprop-p- ethyl } \\
\hline & \multicolumn{2}{|c|}{$\mathrm{P}$} & \multicolumn{2}{|c|}{$\mathrm{L}$} & \multicolumn{2}{|c|}{$\mathrm{P}$} & \multicolumn{2}{|c|}{$\mathrm{L}$} & \multicolumn{2}{|r|}{$\mathrm{P}$} & \multicolumn{2}{|c|}{$\mathrm{L}$} \\
\hline & $\mathrm{R}$ & $S$ & $\mathrm{R}$ & $S$ & $\mathrm{R}$ & $S$ & $\mathrm{R}$ & $S$ & $\mathrm{R}$ & $S$ & $\mathrm{R}$ & $S$ \\
\hline $0,5 X$ & 75,00 & 80,00 & 81,25 & 80,0 & 70,0 & 78,00 & 70,00 & 71,25 & 0,0 & 96,50 & 53,75 & 80,00 \\
\hline $1,0 \mathrm{X}$ & 93,75 & 94,37 & 88,75 & 92,5 & 57,5 & 78,25 & 73,75 & 91,25 & 10,0 & 98,25 & 56,25 & 92,00 \\
\hline $2,0 \mathrm{X}$ & 95,00 & 96,25 & 96,25 & 100,0 & 70,0 & 72,62 & 80,75 & 100,0 & 10,0 & 99,50 & 71,25 & 96,00 \\
\hline $4,0 \mathrm{X}$ & 100,0 & 97,50 & 100,0 & 100,0 & 80,0 & 91,25 & 78,75 & 100,0 & 20,0 & 99,50 & 92,50 & 100,0 \\
\hline $8,0 \mathrm{X}$ & 100,0 & 98,75 & 100,0 & 100,0 & 87,5 & 93,75 & 91,25 & 100,0 & 42,5 & 99,00 & 98,75 & 100,0 \\
\hline
\end{tabular}


Pelas curvas de resposta às doses (Figura 1), observa-se que o biótipo suspostamente resistente precisa de uma dose mais elevada de haloxyfop-methyl para a obtenção do mesmo controle alcançado no biótipo suscetível, em ambos o locais estudados. Os valores de $\mathrm{GR}_{50}$ obtidos no experimento de Piracicaba foram $325 \mathrm{e}$ inferior a $60 \mathrm{~g} / \mathrm{ha}$ para o biótipo com suspeita de resistência e para o biótipo suscetível, respectivamente (Tabela 3). Em Londrina, esses valores foram inferiores a menor dose aplicada (60 g/ha) para ambos os biótipos estudados. Biótipos de azevém (Lolium rigidum) apresentaram polarização reduzida nas membranas celulares na presença do herbicida haloxyfopmethyl e, quando as moléculas do produto eram removidas, ocorria uma nova polarização da membrana somente nos biótipos resistentes (Häusler et al.,1991).

As respostas no controle do capimmarmelada em função das doses de fluazifop-pbutil, nos dois locais estudados, são apresentadas na Figura 2. Esse herbicida, aplicado na dose recomendada, não controlou o biótipo com suspeita de resistência (percentagem de controle de $0 \%$ ). Entretanto, a percentagem de controle obtida pelo biótipo suscetível foi $96 \%$ nas condições de Piracicaba (Tabela 2). Em Londrina, foi obtida percentagem média de controle em torno de $21 \%$ para o biótipo B-196 e $83 \%$ para aquele considerado suscetível, utilizando-se a dose recomendada desse herbicida. Os valores de $\mathrm{GR}_{50}$ para esse produto, obtidos a partir do modelo ajustado foram 804 e $713 \mathrm{~g} / \mathrm{ha}$ para o biótipo B196, nos experimentos conduzidos em Piracicaba e Londrina, respectivamente (Tabela 3).

Para o biótipo suscetível, esses valores foram inferiores a menor dose utilizada $(94 \mathrm{~g} / \mathrm{ha})$. Populações de capim colchão (Digitaria sanguinalis), resistentes ao fluazifop-p-butil, foram identificadas no Estado de Wisconsin (Wiederholt \& Stoltenberg, 1995). Verificaram que o biótipo resistente foi 59 vezes mais tolerante a esse produto do que aquele considerado como suscetível. Populações de sorgo-de-alepo (Sorghum halepense), resistentes ao fluazifop-p- butil, foram estudadas em condições de casa-devegetação por Smeda et al. (1997). Os valores de $\mathrm{R} / \mathrm{S}$ obtidos foram superiores a 388 , baseando-se no crescimento de rizomas da planta. Um biótipo de capim pé-de-galinha (Eleusine indica), também resistente a esse herbicida, foi avaliado por Marshall et al. (1994). Verificaram que o biótipo sobreviveu quando exposto a, até mesmo, 4000 $\mathrm{g} / \mathrm{ha}$ do produto.

Quando foi aplicada a dose recomendada do herbicida sethoxydim, a percentagem média de controle para o biótipo com suspeita de resistência foi $0 \%$ e 5\% para os experimentos de Piracicaba e Londrina, respectivamente (Tabela 2). Entretanto, para a mesma dose, os valores obtidos foram $99 \mathrm{e}$ 91\% no biótipo suscetível, nos dois locais estudados. As respostas no controle do capim marmelada, em função das doses do sethoxydim, são apresentadas na Figura 3. Foi observado que o biótipo B-196 apresentou elevado grau de resistência ao sethoxydim quando comparado ao biótipo suscetível. Os valores de $\mathrm{GR}_{50}$ foram superiores a maior dose utilizada ( $1840 \mathrm{~g} / \mathrm{ha})$ para o biótipo com suspeita de resistência e, para o biótipo suscetível, esse valor foi menor que a menor dose utilizada $(115 \mathrm{~g} / \mathrm{ha})$, em ambos os locais estudados (Tabela 3). Quatro populações de Avena fatua, procedentes do oeste do Canadá, foram expostas a aplicações repetidas de sethoxydim durante 10 anos (Heap et al., 1993). Observaram que plantas dessa invasora escapavam à ação de herbicidas dos grupos químicos das ciclohexanodionas e ariloxifenoxipropionatos. Uma das populações estudadas recebeu uma aplicação 150 vezes maior do que a população suscetível para obter $50 \%$ de redução da matéria seca da parte aérea.

As respostas no controle de capimmarmelada em função das doses dos herbicidas clethodim e propaquizafop são apresentadas nas Figuras 4 e 5. Em relação a esses dois herbicidas, foram obtidos valores médios de percentagem de controle superiores a $70 \%$ com a aplicação da menor dose dos produtos para os biótipos avaliados nos dois experimentos (Tabela 2). 

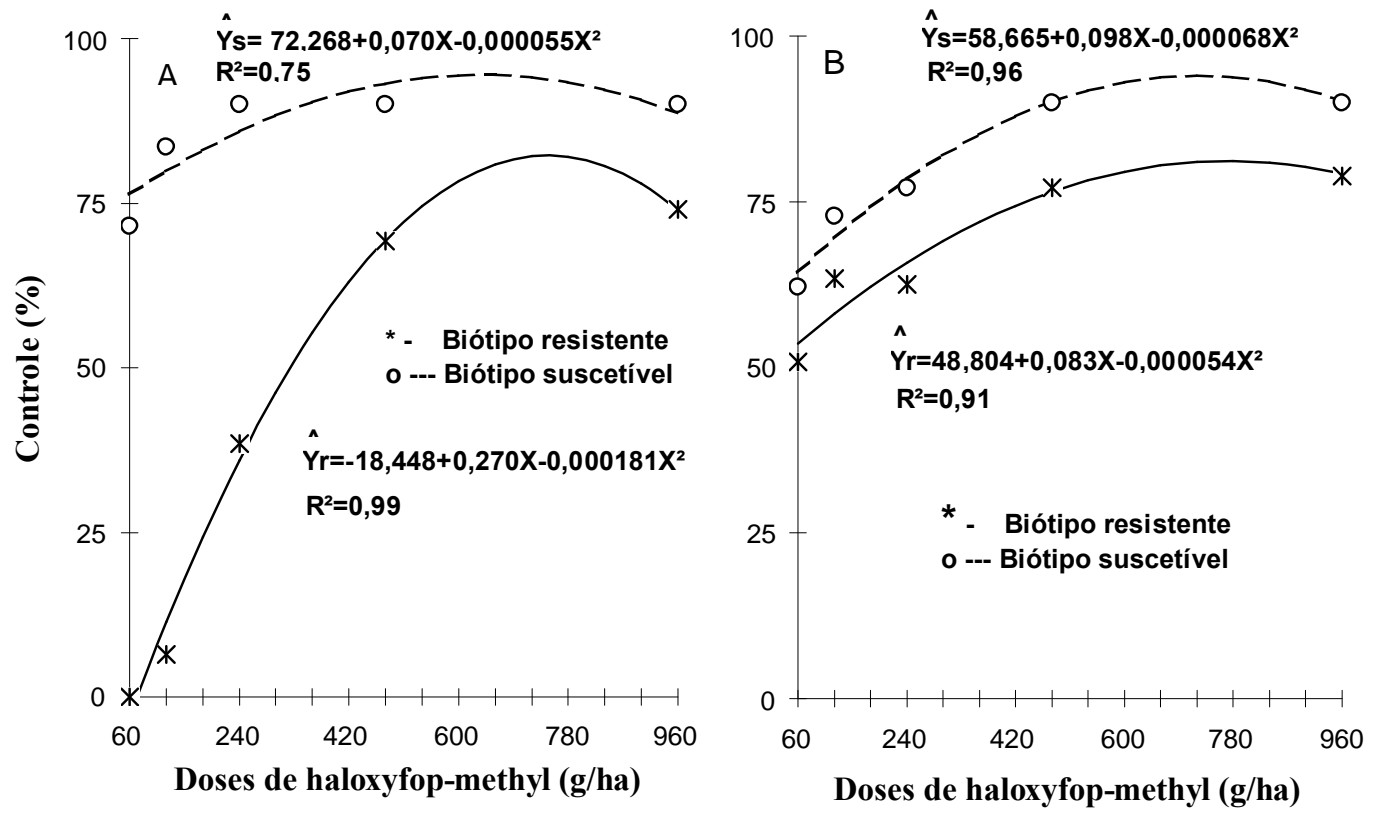

FIGURA 1. Curvas de resposta do controle do capim-marmelada, em função das doses do herbicida haloxyfop-methyl, nos experimentos conduzidos em Piracicaba (A) e Londrina (B).
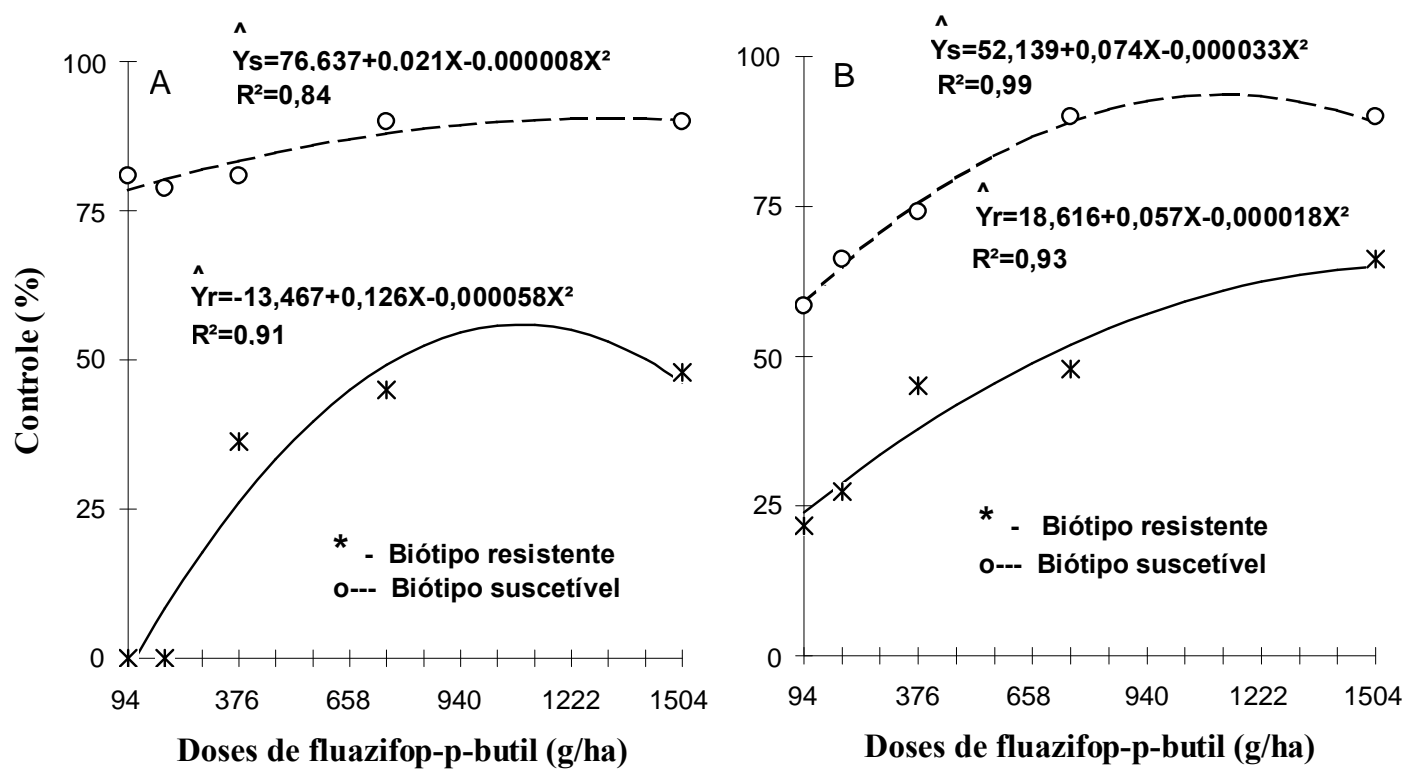

FIGURA 2. Curvas de resposta do controle do capim-marmelada, em função das doses do herbicida fluazifop-p-butil, nos experimentos conduzidos em Piracicaba (A) e Londrina (B). 

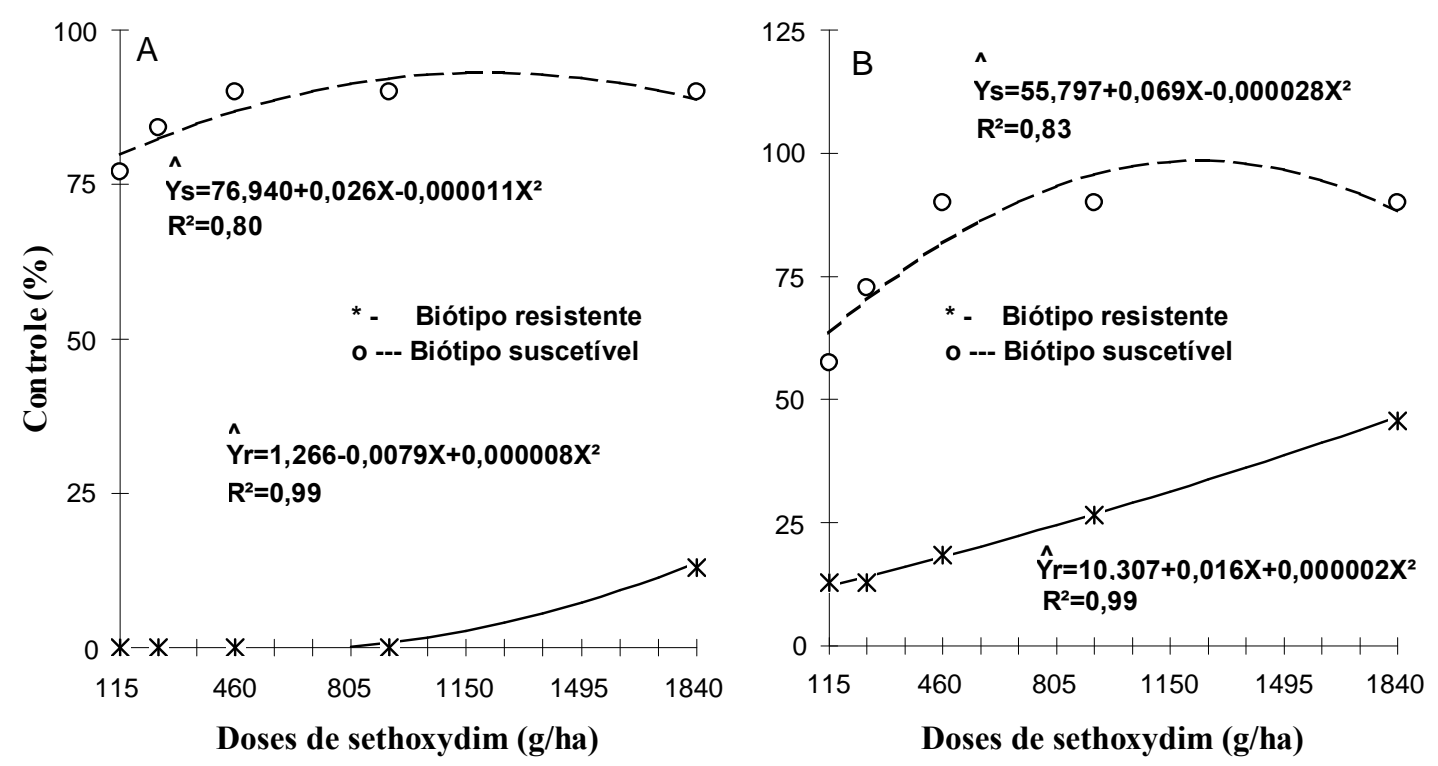

FIGURA 3. Curvas de resposta do controle do capim-marmelada, em função das doses do herbicida sethoxydim, nos experimentos conduzidos em Piracicaba (A) e Londrina (B).
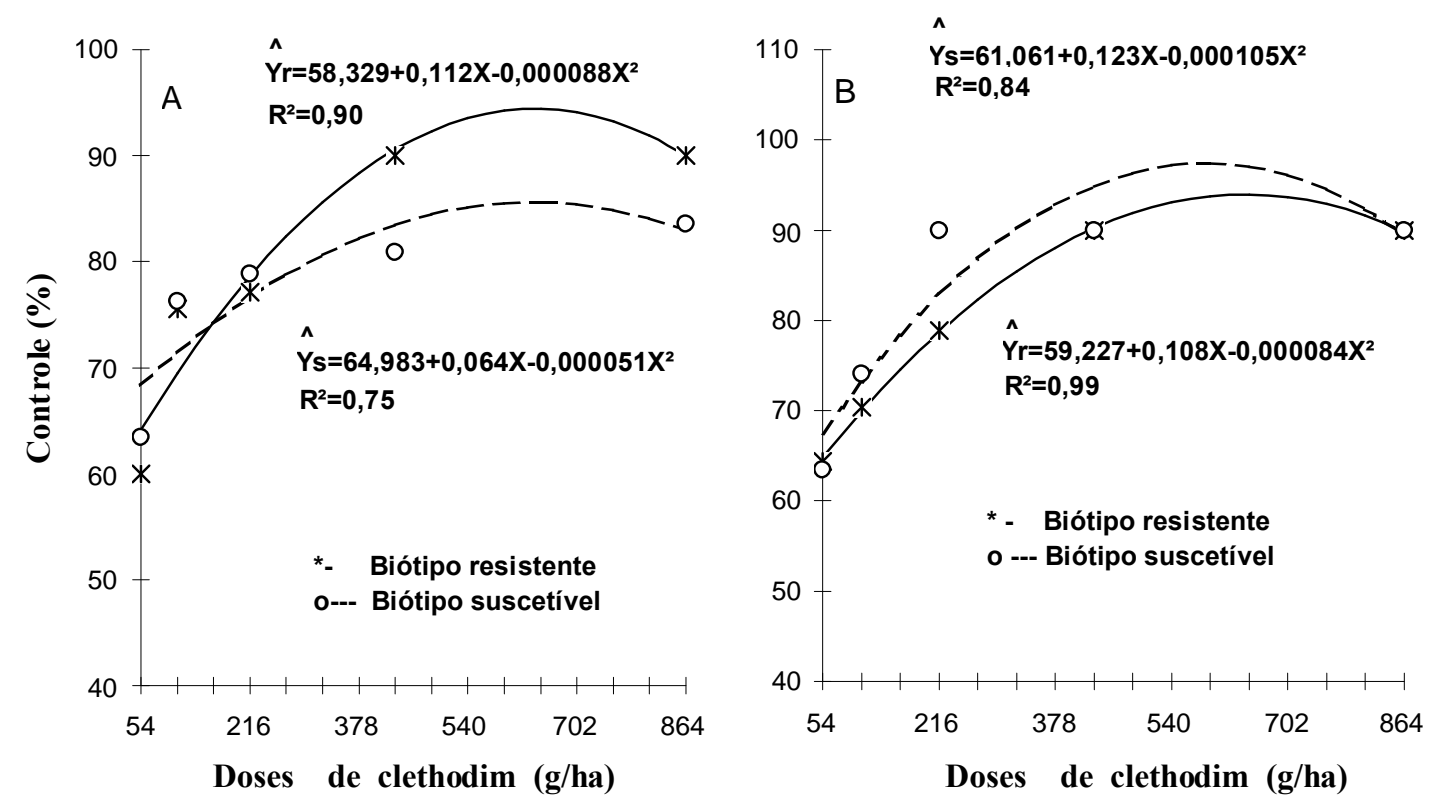

FIGURA 4. Curvas de resposta do controle do capim-marmelada, em função das doses do herbicida clethodim, nos experimentos conduzidos em Piracicaba (A) e Londrina (B). 


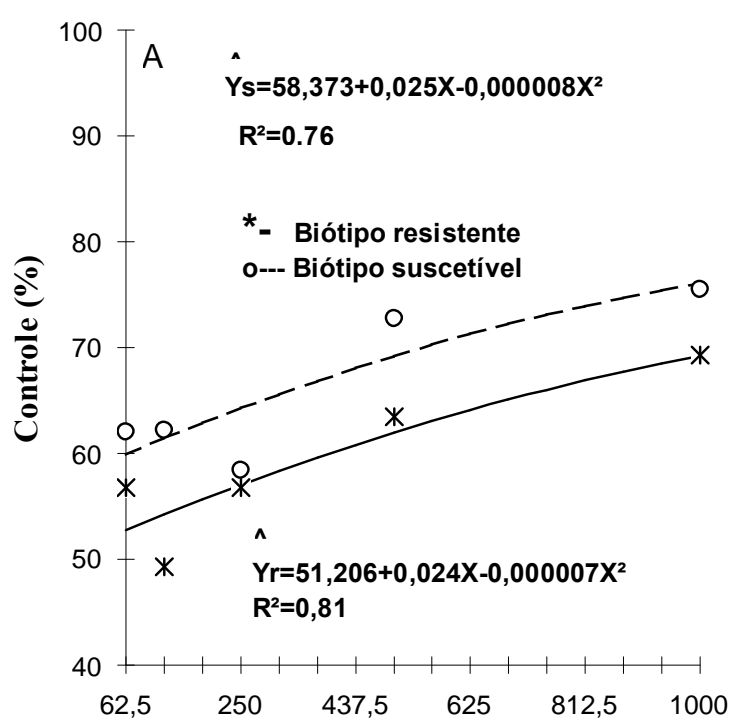

Doses de propaquizafop (g/ha)

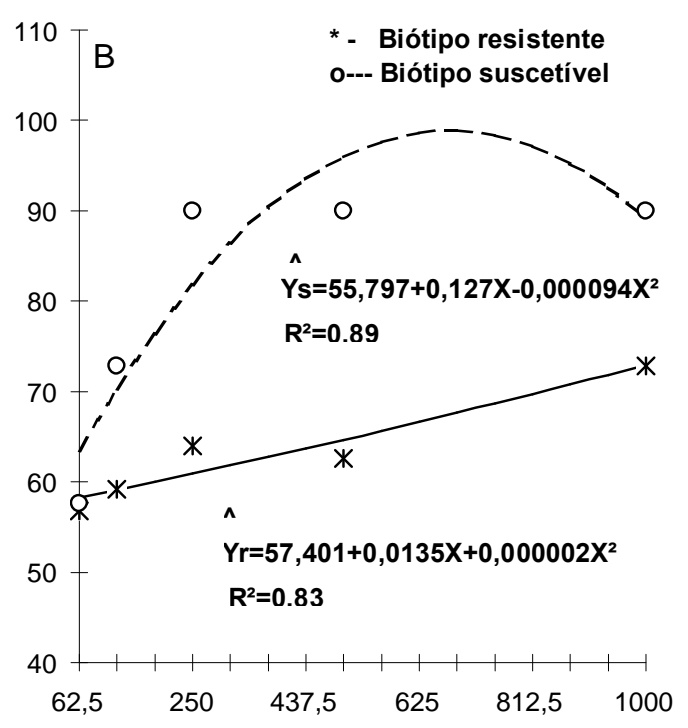

Doses de propaquizafop $(\mathrm{g} / \mathrm{ha})$

FIGURA 5. Curvas de resposta do controle do capim-marmelada, em função das doses do herbicida propaquizafop, nos experimentos conduzidos em Piracicaba (A) e Londrina (B).
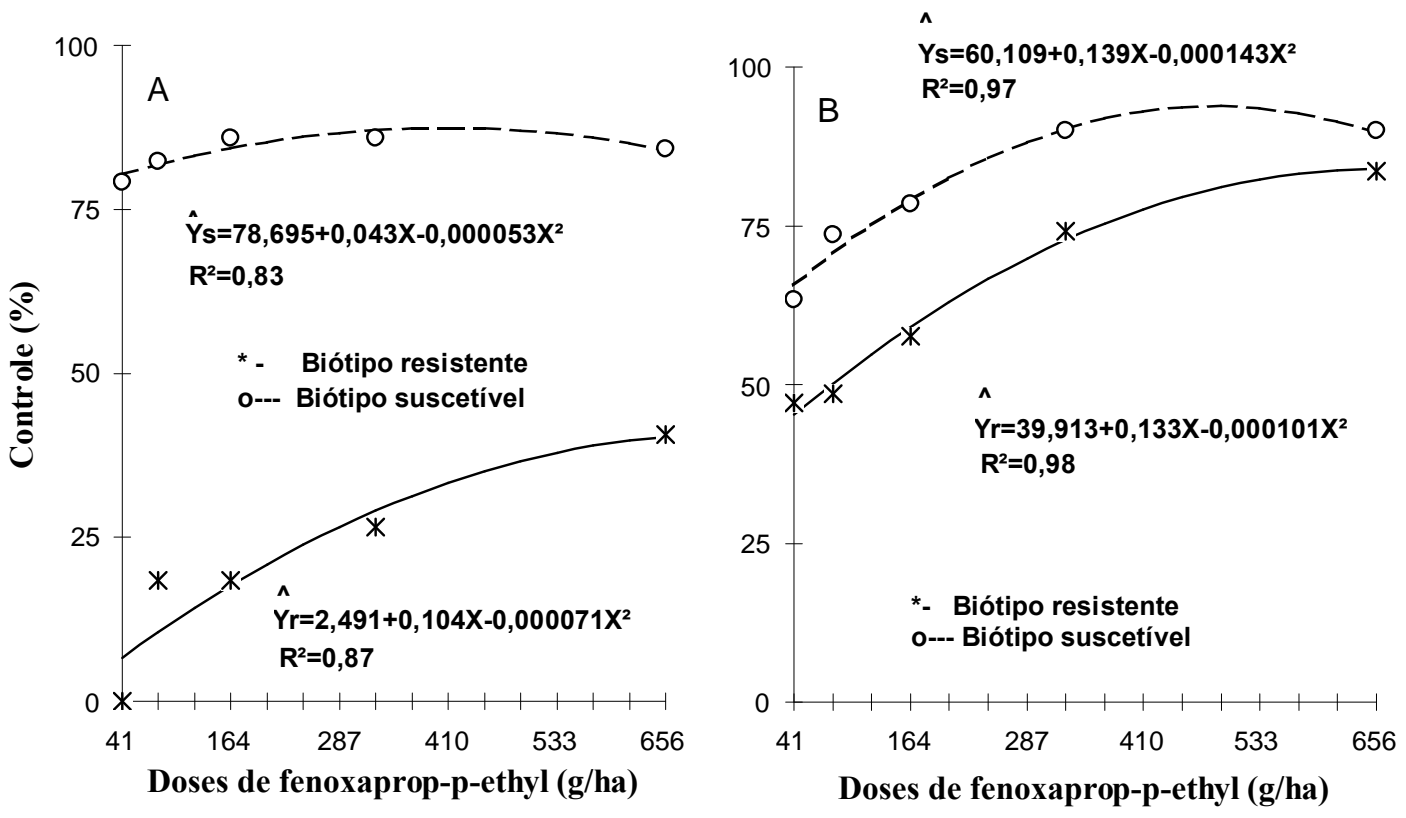

FIGURA 6. Curvas de resposta do controle do capim-marmelada, em função de doses do herbicida fenoxaprop-p-ethyl, nos experimentos conduzidos em Piracicaba (A) e Londrina (B). 
A dose necessária para se obter $50 \%$ de controle está abaixo da menor dose aplicada (54 e $62 \mathrm{~g} / \mathrm{ha}$ ) de ambos os produtos e biótipos nos dois locais estudados (Tabela 3).

Nas condições de Piracicaba, o herbicida fenoxaprop-p-ethyl aplicado na dose recomendada proporcionou percentagem média de controle correspondente a 10 e $98 \%$ do capimmarmelada supostamente resistente, e daquele considerado suscetível, respectivamente (Tabela 2). No experimento conduzido em Londrina, obteve-se controle de 56\% (biótipo B-196) e $92 \%$ (biótipo suscetível) para a mesma dose. As respostas no controle do capim-marmelada em função das doses de fenoxaprop-ethyl são apresentadas na Figura 6 . O valor de $\mathrm{GR}_{50}$ foi superior a maior dose aplicada (656 g/ha) para o capim-marmelada B-196 e inferior a menor dose utilizada para o biótipo considerado suscetível no experimento conduzido em Piracicaba (Tabela 3).

Em Londrina, o $\mathrm{GR}_{50}$ para o biótipo supostamente resistente foi $84 \mathrm{~g} / \mathrm{ha}$, e para o biótipo suscetível, foi inferior à menor dose aplicada (41 g/ha).
Os valores da relação R/S são apresentados na Tabela 3. Esse parâmetro não foi um bom determinante para diferenciação da resistência, pois, para maioria dos herbicidas testados, a amplitude de doses não foi suficiente para determinar o $\mathrm{GR}_{50}$. Porém, os diferenciais de controle nas doses recomendadas (S-R) apresentados pelos dois biótipos reforça o fato de haver um diferencial de resistência para os herbicidas estudados. Esses valores foram 97 e 11; 96 e 62; 99 e 86; 0,6 e 4; 20 e 17; 88 e 35 para os herbicidas haloxyfop-methyl, fluazifopp-butil, sethoxydim, clethodim, propaquizafop e fenoxaprop-p-ethyl em Londrina e Piracicaba, respectivamente (Tabela 4 ).

\section{AGRADECIMENTOS}

Aos colegas Celso Wobeto (FAPA) e Cleber D. G. Maciel (estagiário Embrapa/UEM), pelo apoio na execução dos experimentos, e aos técnicos agrícolas da Embrapa Soja Mário Nakano e Reinaldo Teruhico Moriyama pela colaboração.

TABELA 3. Doses ( $\mathrm{g} / \mathrm{ha}$ ) correspondente ao $\mathrm{GR}_{50}$ do biótipo resistente (R) e do biótipo suscetível (S) e a relação R/S para o experimento conduzido em Piracicaba e em Londrina.

\begin{tabular}{|c|c|c|c|c|c|c|c|c|}
\hline \multirow{2}{*}{ HERBICIDAS } & \multicolumn{4}{|c|}{ PIRACICABA } & \multicolumn{4}{|c|}{ LONDRINA } \\
\hline & $\mathrm{R}$ & $S$ & & $\mathrm{R} / \mathrm{S}$ & & $\mathrm{R}$ & $S$ & $\mathrm{R} / \mathrm{S}$ \\
\hline haloxyfop-methyl* & 325,96 & $<60,00$ & $>$ & 5,43 & $<$ & 60,00 & $<60,00$ & - \\
\hline fluazifop-p-butil & 804,31 & $<94,00$ & $>$ & 8,55 & & 713,88 & $<94,00$ & $>7,59$ \\
\hline sethoxydim & $>1840,00$ & $<115,00$ & & 16,00 & & 840,00 & $<115,00$ & $>16,00$ \\
\hline clethodim* & $<\quad 54,00$ & $<54,00$ & & - & $<$ & 54,00 & $<54,00$ & - \\
\hline propaquizafop* & $<\quad 62,50$ & $<62,50$ & & - & $<$ & 62,50 & $<62,50$ & - \\
\hline fenoxaprop-p-ethyl & $>656,00$ & $<41,00$ & $>$ & 16,00 & & 84,15 & $<41,00$ & $<2,05$ \\
\hline
\end{tabular}

* não foi possível determinar a relação R/S. 
TABELA 4. Percentagem de controle obtido na dose recomendada dos herbicidas e o diferencial de controle (S-R) dos biótipos estudados nos experimentos conduzidos em Piracicaba e Londrina.

\begin{tabular}{|c|c|c|c|c|c|c|}
\hline \multirow{2}{*}{ HERBICIDAS } & \multicolumn{3}{|c|}{ PIRACICABA } & \multicolumn{3}{|c|}{ LONDRINA } \\
\hline & $\mathrm{R}$ & $S$ & S-R & $\mathrm{R}$ & $S$ & S-R \\
\hline haloxyfop-methyl & 1,25 & 98,75 & 97,50 & 80,00 & 91,25 & 11,25 \\
\hline fluazifop-p-butil & 0,00 & 96,25 & 96,25 & 21,25 & 83,75 & 62,50 \\
\hline sethoxydim & 0,00 & 99,00 & 99,00 & 5,00 & 91,25 & 86,25 \\
\hline clethodim & 93,75 & 94,37 & 0,62 & 88,75 & 92,50 & 3,75 \\
\hline propaquizafop & 57,50 & 78,25 & 20,75 & 73,75 & 91,25 & 17,50 \\
\hline fenoxaprop-p-ethyl & 10,00 & 98.25 & 88,25 & 56,25 & 92,00 & 35,75 \\
\hline
\end{tabular}

\section{LITERATURA CITADA}

BLAIR, W. Herbicide resistance in weeds: an overview. American Cyanamid, Agricultural Division, Crop Protection Chemicals Department. Princeton, New Jersey; 16 p.,1991.

CHRISTOFFOLETI, P. J.; PONCHIO, J. A. R.; BERG, E. V. D.; VICTÓRIA FILHO, R. Imidazolinone resistant Bidens pilosa byotipes in the Brazilian soybean areas. Meeting of the Weed Science Society of America. Norfolk. WSSA Abstract. v.36, p.10, 1996.

CHRISTOFFOLETI, P. J.; CORTEZ, M.G.; VICTÓRIA FILHO, R. Resistance of alexanderweed (Brachiaria plantaginea) to ACCase inhibitor herbicides in soybean from Paraná State-Brazil. Meeting of the Weed Science Society of America. Chicago. WSSA Abstract. v.38, p.65, 1998.

GAZZIERO, D. L. P.; CHRISTOFFOLETI, P. J.; MACIEL, C. D. M.; SCARAMUZZA Jr.; J. R. Resistência de biótipos de Brachiaria plantaginea aos herbicidas inibidores da ACCase aplicados em soja. XXI Congresso
Brasileiro da Ciência das Plantas Daninhas. Caxambu-MG, Resumos...p.88, 1997.

HÄUSLER, R. E.; HOLTUM, J. A. M.; POWLES, S. B. Cross-resistance to herbicides in annual ryegrass (Lolium rigidum). IV Correlation between membrane effects and resistance to graminicides. Plant Physiol., v.97, n.3, p. 1035-1043, 1991.

HEAP, I. M.; MURRAY, B. G.; LOEPPKY, H. A.; MORRISON, I. N. Resistance to aryloxyphenoxypropionate and cyclohexanedione herbicides in wild oat (Avena fatua). Weed Sci.; v. 41, p.232-238, 1993.

MARSHALL, G.; KIRKWOOD, R. C.; LEACH; G. E. Comparative studies on graminicideresistant and susceptable biotypes of Eleusine indica. Weed Res., v.34, n.3, p. 177-185, 1994.

MAXWELL, B. D.; ROUSH, M. L.; RADOSEVICH, S. R. Predicting the evolution and dynamics of herbicide resistance in weed populations. Weed Tech., v. 4, p.2-13, 1990. 
MUDGE, L. C.; GOSSETT, B. J.; MURPHY, T. R. Resistance of goosegrass (Eleusine indica) to dinitroaniline herbicides. Weed Sci., v.32, p.591-594, 1984.

PONCHIO, J. A. R. Resistência de Bidens pilosa L. aos herbicidas inibidores da enzima acetolactato sintase. Piracicaba - ESALQ, 1997. 120p.(Tese DS).

POWLES, S. B.; PRESTON, C. Herbicide cross resistance and multiple resistance in plants. Department of Crop Protection. Waite Agricultural Research Institute, South Australia. University of Adelaide. 34 p., 1995.

RYAN, G. F. Resistance of common groundsel to simazine and atrazine. Weed Sci., v.18, p.614-616, 1970.

SMEDA, R. J.; SNIPES, C. E.; BARRENTINE, W. L. Identification of graminicide-resistant johnsongrass (Sorghum halepense). Weed Sci., v. 45, n. 1, p. 132-137, 1997.

VIDAL, R. A.; FLECK, N. G.; THEISEN, G.; NEVES, R.; PETRY, L. A. Picão-preto e leiteira resistentes aos inibidores da ALS não apresentam resistência aos herbicidas com diferentes mecanismos de ação. XXI Congresso Brasileiro da Ciência das Plantas Daninhas. Caxambu-MG, Resumos...p.465, 1997 a.

VIDAL, R.A.; OLIVEIRA, N. A.; FLECK, N. G.; GUIMARÃES, F. B.; SILVA, N. G. Misturas de mecanismos de ação de herbicidas no controle de leiteira resistente aos inibidores da ALS. XXI Congresso Brasileiro da Ciência das Plantas Daninhas. Caxambu-MG, Resumos...p.468, 1997 b.

VIDAL, R. A.; FLECK, N. G. Three weed species with confirmed resistance to herbicides in Brazil. Meeting of the Weed Science Society of America. WSSA Abstract. v.37, p.100, 1997.

WHITEHEAD, C. W.; SWITZER, C. M. The differencial response of strains of wild carrot to 2,4-D and related herbicides. Can. J. Plant Sci., v.43, p.255-262, 1962.

WIEDERHOLT, R. J.; STOLTENBERG， D. Cross resistance of a large crabgrass (Digitaria sanguinalis) accession to aryloxyphenoxypropionate and ciclohexanodione herbicides. Weed Tech. v.9, n.3, p. 518-524, 1995. 